\title{
Um modelo integrado para a programação de voos e alocação de frotas
}

\author{
Daniel Jorge Caetano ${ }^{1}$ e Nicolau Dionísio Fares Gualda ${ }^{2}$
}

\begin{abstract}
Resumo: Este artigo apresenta um modelo para a otimização da malha a ser atendida por uma empresa aérea, resolvendo, de forma integrada, os problemas de programação de voos e alocação de frotas. O modelo inclui restrições operacionais específicas como a existência de slots de pouso e decolagem e a limitada flexibilidade de opções de aeroportos oriunda de um tráfego composto primariamente por passageiros. O modelo foi testado e aplicado com sucesso a um caso de uma empresa aérea regional brasileira, resultando em uma programação de voos completa e provendo informações de suporte à decisão sobre a possibilidade de novos voos ou o uso de outros tipos de aeronave.
\end{abstract}

Palavras-chave: transporte aéreo; alocação de frotas, programação de voos, programação linear.

\begin{abstract}
This paper proposes a model to optimize the network to be served by an airline, solving, in an integrated way, the flight schedule definition and the fleet assignment problems. The model includes specific operational constraints like takeoff and landing slots and limited flexibility on airport options due to a traffic mainly composed by passengers. The model was successfully applied to a Brazilian regional airline case, which resulted in a complete schedule and provided information to support decisions on new possible flights or utilization of other aircraft type.

Keywords: air transportation; fleet assignment; flight scheduling; linear programming.
\end{abstract}

\section{INTRODUÇÃO}

Com o crescimento da demanda de passageiros ao longo dos anos, houve um aumento na frequência dos voos, com uma ligeira queda no número de assentos por voo (Swan, 2002). Este fato, aliado à crescente competição entre as empresas aéreas, torna necessária a utilização de modelos que permitam definir novas malhas aéreas de acordo com a evolução da demanda, visando a uma redução de custos operacionais (Klabjan, 2004). Tais problemas são, entretanto, relativamente complexos, com um grande número de variáveis e restrições (Hane et al., 1995; Klabjan, 2004), tradicionalmente levando a uma divisão do problema em etapas, com modelos mais simples, específicos para cada uma delas (Rabetanety et al. 2006).

No Brasil, o problema tem características específicas como, por exemplo, a existência de restrições operacionais em aeroportos relevantes (CGNA, 2009; MD, 2008; Caetano e Gualda, 2008), sendo a maior parte deste tráfego de passageiros (Anuário, 2007 apud Oliveira, 2009), dificultando a adoção de aeroportos alternativos aos saturados.

Nesse contexto, este trabalho apresenta um modelo que trata de maneira integrada duas das etapas do planejamento operacional de uma empresa aérea: a Programação de Vôos (Schedule Generation Problem) e o problema da Alocação de Frota (Fleet Assignment Problem), aplicando-o para o caso de uma empresa aérea operando no mercado doméstico brasileiro.

Inicialmente é apresentada uma breve revisão dos conceitos envolvidos na solução desses problemas, seguida da

\footnotetext{
${ }^{1}$ Daniel Jorge Caetano, Departamento de Engenharia de Transportes, Escola Politécnica, Universidade de São Paulo, São Paulo, SP, Brasil. (e-mail: daniel@caetano.eng.br).

${ }^{2}$ Nicolau Dionísio Fares Gualda, Departamento de Engenharia de Transportes, Escola Politécnica, Universidade de São Paulo, São Paulo, SP, Brasil. (e-mail: ngualda@usp.br).

Manuscrito recebido em 28/3/2011 e aprovado para publicação em 6/5/2011. Este artigo é parte de TRANSPORTES v.19, n.2, 2011. ISSN: 2237-1346
} (online). apresentação das características específicas do problema tratado, do modelo proposto e dos resultados de uma análise de robustez deste modelo, além de uma aplicação a instâncias baseadas na malha de uma empresa aérea nacional.

\section{O PLANEJAMENTO OPERACIONAL DE EMPRESAS AÉREAS}

O planejamento operacional de empresas aéreas pode ser dividido em três grandes problemas inter-relacionados: a definição de quais voos serão oferecidos, de quais aeronaves farão quais voos e de quais serão os tripulantes destes voos. Estas etapas são apresentadas na Figura 1.

A definição de voos é o processo em que se define a $a$ genda de voos. Na primeira parte desta etapa, a Geração de Voos, são identificadas as demandas entre pares de cidades e, assim, são definidos os potenciais voos entre pares de aeroportos, respeitando as restrições mais simples dos aeroportos. A segunda parte desta etapa, denominada Programação de Voos, corresponde à determinação de quais voos serão cumpridos e, neste ponto, devem ser respeitadas restrições de operação mais específicas de cada aeroporto, como horários fixos disponíveis para uma dada empresa aérea, os chamados slots (ANAC, 2008; Klabjan, 2004; Rabetanety et al., 2006).

A alocação de aeronaves é o processo em que se define qual aeronave executará cada voo. Na primeira parte desta etapa, a Alocação de Frotas, é definido, para cada voo, qual o melhor tipo de aeronave para cumpri-lo, visando à maximização da rentabilidade. Na segunda parte desta etapa, a Atribuição de Aeronaves, são definidas as aeronaves específicas para cada voo, respeitando períodos de revisão, de manutenção, dentre outros (Abara, 1989; Barnhart et al., 2003; Klabjan, 2004; Rabetanety et al., 2006).

A alocação de tripulantes é o processo em que se define qual tripulante será escalado para qual aeronave. Na primeira parte, a Definição de Viagens, são definidas as sequências de voos que respeitem as legislações trabalhistas e cri- 


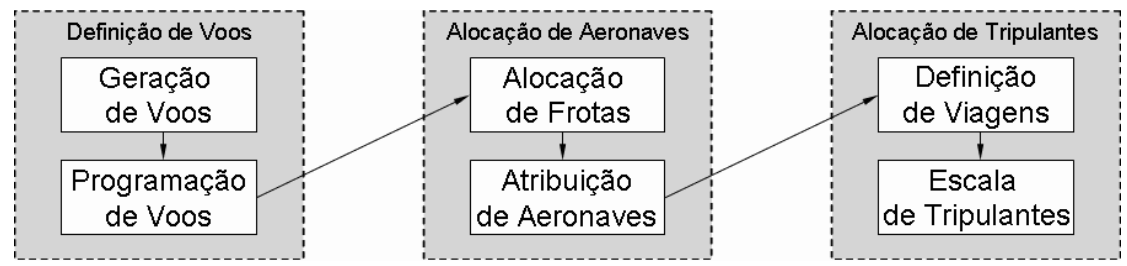

Figura 1. Etapas do Planejamento Operacional de Empresas Aéreas

térios técnicos, que são denominadas viagens. Na segunda etapa, a Escala de Tripulantes, são definidos, finalmente, quais os tripulantes que cumprirão cada uma das viagens. (Barnhart et al., 2003; Gomes e Gualda, 2008; Klabjan, 2004; Rabetanety et al., 2006).

$\mathrm{O}$ aumento da complexidade dos sistemas aéreos e a crescente competitividade levaram à necessidade de criar modelos que incluam um número cada vez maior de rotas e restrições. Tais modelos de grande escala são um grande desafio computacional, pois vários deles são considerados do tipo NP-difícil (Hane et al., 1995). Esta característica se agrava quando são utilizados modelos que solucionam simultaneamente duas ou mais etapas do problema, buscando-se uma otimização global (Klabjan, 2004; Sherali et al., 2006; Rabetanety et al., 2006).

Adicionalmente, a malha definida por esses modelos nem sempre pode ser considerada como uma solução permanente. Quando parte significativa do tráfego de um país de tamanho continental é composta por passageiros, incluindo turistas, a demanda provavelmente sofrerá variações ao longo do ano, alterando a lucratividade de cada voo. Nesse contexto, o mercado regional pode representar um grande desafio para as empresas aéreas, demandando ajustes periódicos em sua malha de voos. Considerando-se ainda que, usualmente, as malhas de empresas regionais são compostas por trechos curtos, há maior facilidade para a atuação de empresas de baixo custo (low cost-low fare), favorecendo a competição e causando uma pressão pela redução das tarifas (Oliveira, 2009). Essa situação pode ser agravada pela concorrência de modos de transporte potencialmente mais econômicos, como o ferroviário e o rodoviário, o que torna importante o constante aperfeiçoamento da malha para a manutenção da competitividade do transporte aéreo.

\section{O PROBLEMA TRATADO}

Embora todas as etapas já descritas sejam interdependentes, a Definição de Voos e a Alocação de Aeronaves são duas boas candidatas ao tratamento conjunto, devido ao seu caráter estratégico para a empresa aérea, com consequências diretas nas etapas posteriores, tanto no âmbito tático quanto operacional. Um modelo integrado deste tipo pode fornecer, por exemplo, os impactos na malha aérea de uma empresa oriundos da utilização de uma nova aeronave, visto que o uso de aeronaves com capacidades diferenciadas pode viabilizar ou inviabilizar determinados trechos. Um outro tipo de situação em que tal integração tem grande utilidade é na avaliação de impactos na malha advindos da restrição de operação em aeroportos onde a empresa atua.

Dependendo do tipo de passageiro de um trecho específico, a demanda pode mudar ao longo da semana. Quando o tráfego é composto principalmente por viajantes a negócios, alguns trechos podem ter mais demanda em um sentido às segundas-feiras e, no sentido contrário, mais demanda às sextas-feiras. Para lidar com tal característica, a rede de voos potenciais precisa ser construída para uma semana completa.

\subsection{Características Propostas para o Modelo}

O modelo deve permitir responder questões de cunho estratégico e tático de uma empresa, incluindo a avaliação do uso de diferentes frotas, fornecendo subsídios para avaliar mudanças nas frotas, e a seleção de voos a serem cumpridos para uma frota específica, adequando a oferta de assentos da melhor maneira possível à demanda. A proposta é evitar voos com assentos vazios na mesma proporção em que deve evitar perder passageiros por falta de assentos, sendo a penalidade para estes casos proporcional à duração do voo.

O modelo deve garantir a continuidade da movimentação de aeronaves de cada tipo, que devem iniciar e finalizar o trajeto na mesma cidade e, se necessário e econômico, devem ocorrer voos para reposicionar as aeronaves, além de respeitar o tempo de manutenção mínima entre voos.

A seleção de voos deve respeitar os horários de slots quando estes forem pré-estabelecidos. No caso de aeroportos de operação não-restrita, o modelo deve considerar ilimitado o número de voos que decolam ou pousam em um dado instante, partindo-se do pressuposto que, na prática, é possível acomodar tais voos com pequenos intervalos entre eles. Ainda assim, apenas um tipo de aeronave deve ser atribuído a cada voo, sendo este um requisito clássico para este tipo de problema.

\section{MODELAGEM MATEMÁTICA}

Os modelos tradicionais de alocação de frota são associados a duas abordagens distintas. Uma delas é baseada em redes de interconexões, em que cada nó representa uma conexão possível entre trechos, e o modelo decide quais conexões devem ser realizadas e quais não (Abara,1989; Sherali et al., 2006). Uma outra abordagem é através de uma rede espaço-tempo, em que os nós representam chegadas ou partidas nos aeroportos; os arcos que ligam estes nós representam os trechos entre os aeroportos ou o tempo que as aeronaves ficam em solo entre um trecho e outro (Berge e Hoperstead, 1993 apud Sherali et al., 2006; Hane et al. 1995).

Tais modelos clássicos pressupõem que a programação de voos está previamente definida e que todos os voos da programação devem ser cobertos e, tradicionalmente, não contemplam restrições de operação nos aeroportos. Para superar estas limitações, faz-se necessário contar com um modelo mais abrangente. $\mathrm{O}$ modelo proposto está baseado no conceito da modelagem espaço-tempo (Berge e Hoperstead, 1993 apud Sherali et al., 2006; Hane et al. 1995), estendido para atender aos requisitos descritos no item 3 , conforme a representação da Figura 2. 


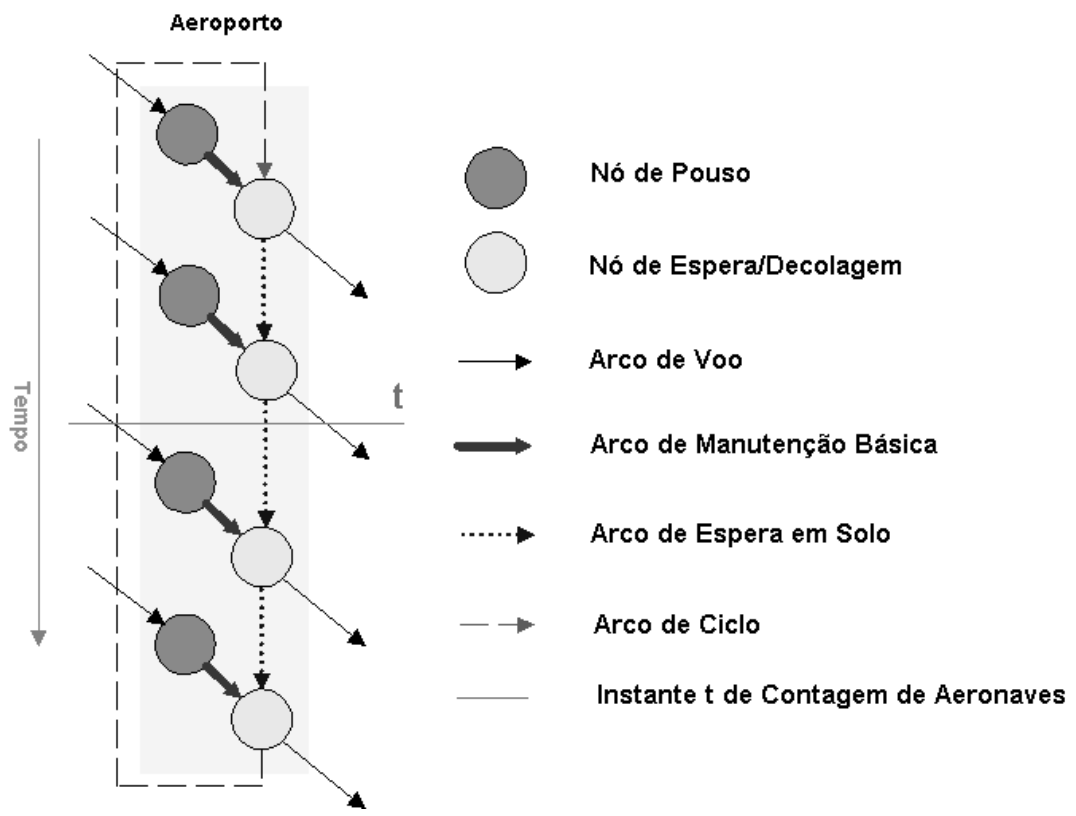

Figura 2. Representação de nós e arcos utilizados para a modelagem

A representação dos momentos de pouso e de espera/decolagem em nós diferentes permite a consideração precisa dos horários dos slots de pouso, diferentemente da representação tradicional em que o tempo de manutenção básica (abastecimento, troca de tripulação e passageiros etc.) é somado ao tempo de duração do arco de voo. Adicionalmente, como os tempos de manutenção podem ser relativamente variados, de acordo com o tipo de aeronave, cada frota é representada por uma rede diferente, com arcos coincidentes para representar os mesmos trechos de voo. O modelo construído com base nessa representação de rede é apresentado a seguir.

$$
[\operatorname{Min}] \sum_{f \in F} \sum_{(i . j) \in L v}\left(D_{i j}-C^{f}\right)^{2} T_{i j} x_{i j}^{f}+\sum_{(i . j) \in L v d} D_{i j}^{2} T_{i j} y_{i j}
$$

Sujeito a:

$$
\begin{gathered}
\sum_{f \in F} x_{i j}^{f}+y_{i j}=1 \quad \forall(i, j) \in L v d \\
\sum_{o \mid(o . k) \in L} x_{o k}^{f}-\sum_{d \mid(k . d) \in L} x_{k d}^{f}=0 \quad \forall k \in N, \forall f \in F \\
\sum_{(i, j) \in L t} x_{i j}^{f} \leq A_{f} \quad \forall f \in F \\
\sum_{f \in F} \sum_{d \mid(i, d) \in L v} x_{i d}^{f} \leq 1 \quad \forall i \in N r d \\
\sum_{f \in F} \sum_{o \mid(o, j) \in L v} x_{o j}^{f} \leq 1 \quad \forall j \in N r a
\end{gathered}
$$

Variáveis binárias:

$$
\begin{aligned}
& x_{i j}^{f} \text { para } \forall(i, j) \in L v d \\
& y_{i j} \text { para } \forall(i, j) \in L v d
\end{aligned}
$$

Variáveis inteiras:

$$
x_{i j}^{f} \geq 0 \text { para } \forall(i, j) \in L \backslash L v d
$$

em que,

$F$ : conjunto de todos os tipos de aeronave, indexados por $f$

$N$ : conjunto de todos os nós do sistema, indexados por $i, j, o, d$ ou $k$, representando um aeroporto e um horário;

$L:$ conjunto dos arcos que representam movimentos de aeronave, em voo, manutenção, em solo ou de retorno, indexados por $(i, j)$, sendo $i$ o nó origem e $j$ o nó destino do movimento;

$L v$ : conjunto dos arcos que representam movimentos de voo;

$L v d$ : conjunto dos arcos que representam voos com demanda;

$D_{i j}: \quad$ demanda de passageiros do nó $i$ ao nó $j$;

$C$ : número de assentos da aeronave do tipo $f$;

$T_{i j}$ : tempo de voo do nó $i$ ao nó $j$;

$x_{i j}^{f}$ : número de aeronaves do tipo $f$ passando pelo arco $(i, j)$

$y_{i j}$ : variável binária que assume o valor 1 quando o voo de $i$ para $j$ não será realizado e 0 , caso contrário;

$L_{t}$ : conjunto dos arcos cuja origem está em tempo igual ou inferior a $t$ e o destino está em tempo maior que o tempo $t$. O instante de tempo $t$ é fixo e deve ser definido com um horário válido de acordo com o problema;

$A_{f}$ : número de aeronaves do tipo $f$ disponíveis;

$N r d$ : conjunto de nós dos aeroportos com restrições de decolagem; e

Nra: conjunto de nós dos aeroportos com restrições de aterrissagem.

A função objetivo deste modelo (expressão 1) visa à minimização das receitas perdidas, considerando, como proxy da perda de receita, a soma dos momentos de transporte as- 
sociados aos assentos vazios e à demanda não atendida. Tal consideração foi inspirada na relação existente entre o custo operacional de um voo e seu momento de transporte máximo (Swan e Adler, 2006).

O primeiro termo da função objetivo representa a soma desses momentos de transporte associados aos voos atendidos por uma aeronave e o segundo termo representa a soma dos momentos de transporte associados aos voos não realizados, cuja demanda foi totalmente perdida. Como a demanda é associada ao trecho, cada elemento da somatória do primeiro termo representa o momento de transporte perdido pelo excesso ou pela falta de assentos em cada voo realizado; já os elementos da somatória do segundo termo representam o momento de transporte perdido, considerando toda a demanda dos trechos não atendidos.

A adoção da diferença entre a demanda e a capacidade da aeronave em um termo quadrático evita a ocorrência de termos negativos, que tornariam inadequado o comportamento da função objetivo - segundo as premissas desse modelo, a alocação de uma aeronave com capacidade superior à demanda de um trecho é tão indesejada quanto a alocação de uma aeronave com capacidade inferior à demanda do mesmo. Adicionalmente, a duração do voo foi mantida fora desse termo quadrático, para evitar que a ênfase da seleção de voos deixe de ser o equilíbrio entre oferta e demanda e passe a ser a seleção de voos de menor duração.

A restrição representada na expressão 2 garante que, no máximo, uma aeronave cumprirá um voo com demanda. Caso nenhuma aeronave seja alocada a um voo com demanda, a variável $y_{i j}$ será usada para indicar a não cobertura do trecho e a perda total da demanda a ele associada. A presença da variável $y_{i j}$ é importante para a atribuição de uma penalidade para os voos com demanda que não sejam executados, levando à seleção de voos que minimizem o número de passageiros perdidos e de assentos vazios. Essa é a característica que permite a existência de voos opcionais, isto é, o modelo não exige que todos os voos sejam cumpridos, como ocorreria em um modelo de cobertura tradicional; por outro lado, os trechos de voos não cobertos levam a uma penalidade na função objetivo.

A restrição representada na expressão 3 garante o equilíbrio em cada um dos nós de cada frota. A restrição é igual para todos os nós, incluindo os iniciais e finais, o que significa que a rede é cíclica e fechada, não havendo fontes e sorvedouros de aeronaves.

Nessas condições, o número de aeronaves fluindo pela rede é constante em qualquer instante $t$. Assim, é suficiente garantir que o número de aeronaves máximo de cada frota seja respeitado em um único instante $t$, como é representado na expressão 4, através da somatória de aeronaves que atravessam todos os arcos que incluam o instante $t$, para cada frota.

Como há nós específicos para representar os pousos e decolagens, os nós em aeroportos de operação restrita podem representar também os slots. Assim, a restrição representada na expressão 5 garante que apenas um voo parta de um slot de decolagem de um aeroporto de operação restrita e, analogamente, a restrição da expressão 6 garante que apenas um voo pouse em um slot de aterrissagem de um aeroporto restrito.

Como cada voo só pode ser cumprido por uma única aeronave, as variáveis que representam os arcos de voos com demanda e as variáveis que indicam se um voo foi ou não cumprido são binárias, como especificam as expressões 7 e 8. Como os arcos de manutenção e espera podem possuir um fluxo de várias aeronaves, as demais variáveis que representam arcos são inteiras e maiores ou iguais a zero, como especificado na expressão 9.

\section{AMBIENTE DE SOLUÇÃO}

Os dados de entrada devem ser fornecidos através de arquivos texto, que conterão todas as informações necessárias de maneira tabular. Esses arquivos são explicados a seguir.

- Arquivo de Voos Potenciais: deve conter a tabela de voos potenciais e todos os voos cujas demandas são relevantes. Cada voo deve ter sua demanda específica indicada;

- Arquivo de Tempos de Voo: deve indicar os tempos de voo entre os pares de cidades. Se um voo não existir nesta tabela, ele será considerado impossível;

- Arquivo de Aeronaves: deve indicar as aeronaves disponíveis e suas características, incluindo tempo mínimo de manutenção após voo e capacidade da aeronave; e

- Arquivo de Aeroportos: deve indicar os aeroportos cuja operação é restrita. É fundamental a existência de voos de partida e de chegada nestes aeroportos, ainda que suas demandas sejam zero, para demarcar os slots disponíveis.

A transformação desses dados em uma rede completa é feita por um software em Java, desenvolvido para gerar o modelo matemático. A rede de voos potenciais é expandida com os voos de reposicionamento de aeronaves (voos sem demanda, vazios). Os arcos de reposicionamento devem também respeitar as restrições de operação de cada aeroporto.

Após a criação dos arcos de reposicionamento, são criados os arcos de solo, que indicam períodos de espera das aeronaves nos aeroportos. Finalmente, é criado um arco do último ao primeiro nó de um aeroporto, para que o posicionamento final das aeronaves seja igual ao inicial.

Com a rede pronta, o software gera o modelo matemático, criando um arquivo no formato LP (Linear Programming model), padrão adotado pela maioria dos pacotes otimizadores do mercado. Para este trabalho foi selecionado o pacote ILog CPlex 11.0, executado em um computador com processador Intel Core2Quad de $2.4 \mathrm{GHz}$ e $2 \mathrm{~GB}$ de memória RAM.

\section{ANÁLISE DE ROBUSTEZ}

A análise de robustez do modelo foi realizada com base em uma série de testes compostos por situações críticas e cujas soluções são previamente conhecidas.

Os resultados de todos os testes realizados, descritos a seguir, estiveram de acordo com as expectativas, com resultados rápidos e precisos, indicando que o modelo é robusto para lidar com os problemas de malha aérea que se encaixem nas características descritas no item 3 .

\subsection{Melhor escolha de aeronaves para um conjunto de trilhos}

Os primeiros testes visaram à verificação da correta escolha de aeronaves para os diferentes voos pelo modelo. Assim, foram definidos dois trilhos, que são sequências de trechos 
a serem cumpridos por uma aeronave, envolvendo 3 cidades cada um. O primeiro trilho conta com uma demanda de 100 passageiros por trecho e segundo com uma demanda de 116 passageiros por trecho, conforme indicado na Tabela 1. A Tabela 2 indica a duração de todos os voos possíveis.

Tabela 1. Trilhos com demanda contínua - formato: aeroporto (dia - hora)

\begin{tabular}{lll}
\hline Trilho & Demanda & Trilho \\
\hline & & $\mathrm{A}(1-1: 40)-\mathrm{B}(1-7: 40)-\mathrm{A}(1-13: 40)$ \\
1 & 100 & $-\mathrm{C}(1-23: 00)-\mathrm{A}(2-6: 20)$ \\
\hline & & $\mathrm{A}(1-1: 40)-\mathrm{C}(1-11: 00)-\mathrm{B}(1-18: 40)$ \\
2 & 116 & $-\mathrm{C}(2-2: 20)-\mathrm{A}(2-11: 40)$ \\
\hline
\end{tabular}

Tabela 2. Voos possíveis (ambas as direções)

\begin{tabular}{lll}
\hline Aeroporto 1 & Aeroporto 2 & Duração (min) \\
\hline A & B & 300 \\
B & C & 400 \\
A & C & 500 \\
\hline
\end{tabular}

Primeiramente foi informada a disponibilidade de duas frotas, uma para 100 passageiros e outra para 116 passageiros, sendo cada frota composta por 3 aeronaves. O tempo de manutenção obrigatória após voo foi definido como sendo de 45 minutos para ambas as aeronaves.

A geração do modelo demorou cerca de 2 segundos, resultando em um modelo com 540 restrições e 948 variáveis.
Sua resolução pelo CPlex não demorou mais que $0,04 \mathrm{~s}$, e está representada na Tabela 3 , indicando a esperada alocação de uma única aeronave para 100 passageiros para o trilho 1 e de uma única aeronave para 116 passageiros para o trilho 2. As outras aeronaves ficaram em espera e não são apresentadas na tabela.

Convém observar que nenhuma das aeronaves conseguirá cumprir seus voos em dias consecutivos com os trilhos propostos, pois a finalização do último voo ocorre em momento posterior ao que seria necessário para a realização do ciclo. Para gerar soluções que atendam a ciclos diários é necessário gerar soluções para voos para vários dias consecutivos. Uma aplicação deste tipo será vista na seção 7.

Gerando um modelo para o problema anterior, mas definindo uma única aeronave para 100 passageiros, o resultado é um modelo com 274 restrições e 478 variáveis. A alocação, resolvida pelo CPlex em cerca de 0,01s, está apresentada na Tabela 4.

Neste caso, a aeronave para 100 passageiros foi alocada ao trilho 2, que possui uma demanda de 116 passageiros. Este é um comportamento previsível e adequado do modelo em que, quando não for possível atender a toda a demanda, as aeronaves serão alocadas nas posições em que ocorrer a maior demanda, ainda que esta demanda supere a capacidade da aeronave.

Resolvendo o mesmo problema apenas com uma aeronave para 116 passageiros, os resultados são idênticos aos da

Tabela 3. Alocação de frota aos trilhos (incluindo manutenção e espera)

\begin{tabular}{|c|c|c|c|}
\hline Frota (Aeronaves) & Evento & Origem (Dia-Hora) & Destino (Dia - Hora) \\
\hline 100 pax (1) & Voo & $\mathrm{A}(0-01: 40)$ & $\mathrm{B}(0-06: 40)$ \\
\hline $100 \operatorname{pax}(1)$ & Manutenção & $\mathrm{B}(0-06: 40)$ & $\mathrm{B}(0-07: 25)$ \\
\hline $100 \operatorname{pax}(1)$ & Espera & $\mathrm{B}(0-07: 25)$ & $\mathrm{B}(0-07: 40)$ \\
\hline $100 \operatorname{pax}(1)$ & Voo & $\mathrm{B}(0-07: 40)$ & $\mathrm{A}(0-12: 40)$ \\
\hline $100 \operatorname{pax}(1)$ & Manutenção & $\mathrm{A}(0-12: 40)$ & $\mathrm{A}(0-13: 25)$ \\
\hline $100 \operatorname{pax}(1)$ & Espera & $A(0-13: 25)$ & A $(0-13: 40)$ \\
\hline $100 \operatorname{pax}(1)$ & Voo & $\mathrm{A}(0-13: 40)$ & $\mathrm{C}(0-22: 00)$ \\
\hline $100 \operatorname{pax}(1)$ & Manutenção & $\mathrm{C}(0-22: 00)$ & $\mathrm{C}(0-22: 45)$ \\
\hline $100 \operatorname{pax}(1)$ & Espera & $\mathrm{C}(0-22: 45)$ & $\mathrm{C}(0-23: 00)$ \\
\hline $100 \operatorname{pax}(1)$ & Voo & $\mathrm{C}(0-23: 00)$ & $\mathrm{A}(1-07: 20)$ \\
\hline $100 \operatorname{pax}(1)$ & Manutenção & A $(1-07: 20)$ & A $(1-08: 05)$ \\
\hline 116 pax (1) & Voo & $\mathrm{A}(0-01: 40)$ & $\mathrm{C}(0-10: 00)$ \\
\hline 116 pax (1) & Manutenção & $\mathrm{C}(0-10: 00)$ & $C(0-10: 45)$ \\
\hline 116 pax (1) & Espera & $\mathrm{C}(0-10: 45)$ & $\mathrm{C}(0-11: 00)$ \\
\hline $116 \operatorname{pax}(1)$ & Voo & $\mathrm{C}(0-11: 00)$ & $\mathrm{B}(0-17: 40)$ \\
\hline 116 pax (1) & Manutenção & $\mathrm{B}(0-17: 40)$ & $\mathrm{B}(0-18: 25)$ \\
\hline $116 \operatorname{pax}(1)$ & Espera & $\mathrm{B}(0-18: 25)$ & $\mathrm{B}(0-18: 40)$ \\
\hline $116 \operatorname{pax}(1)$ & Voo & $\mathrm{B}(0-18: 40)$ & $\mathrm{C}(1-01: 20)$ \\
\hline $116 \operatorname{pax}(1)$ & Manutenção & $\mathrm{C}(1-01: 20)$ & $C(1-02: 05)$ \\
\hline 116 pax (1) & Espera & $\mathrm{C}(1-02: 05)$ & $\mathrm{C}(1-02: 20)$ \\
\hline $116 \operatorname{pax}(1)$ & Voo & $\mathrm{C}(1-02: 20)$ & A $(1-10: 40)$ \\
\hline 116 pax (1) & Manutenção & A $(1-10: 40)$ & $C(1-11: 25)$ \\
\hline
\end{tabular}

Tabela 4. Alocação de uma aeronave para 100 passageiros (apenas voos)

\begin{tabular}{llll}
\hline Frota (Aeronaves) & Evento & Origem (Dia - Hora) & Destino (Dia - Hora) \\
\hline 100 pax (1) & Voo & A $(0-01: 40)$ & C $(0-10: 00)$ \\
100 pax (1) & Voo & C $(0-11: 00)$ & B $(0-17: 40)$ \\
100 pax (1) & Voo & B $(0-18: 40)$ & C $(1-01: 20)$ \\
100 pax (1) & Voo & C $(1-02: 20)$ & A $(1-10: 40)$ \\
$-(0)$ & Voo & A $(0-01: 40)$ & B $(0-06: 40)$ \\
$-(0)$ & Voo & B $(0-07: 40)$ & A $(0-12: 40)$ \\
$-(0)$ & Voo & A $(0-13: 40)$ & C $(0-22: 00)$ \\
$-(0)$ & Voo & C $(0-23: 00)$ & A $(1-07: 20)$ \\
\hline
\end{tabular}


Tabela 4, com o uso da aeronave para 116 passageiros substituindo a de 100 passageiros no trilho 2 .

\subsection{Criação adequada de voos de reposicionamento}

Nos testes da seção 6.1 não foi necessário realizar nenhum voo além daqueles presentes na tabela de voos potenciais, para que fosse possível cumprir de maneira eficiente os voos desejados. Entretanto, esta nem sempre é uma suposição válida: a solução mais eficiente pode envolver algum reposicionamento de aeronaves.

Os testes aqui propostos objetivam a verificação da adequada utilização de tais voos de reposicionamento. Assim, foram definidos três voos na tabela de voos potenciais, indicados na Tabela 5, sendo os voos possíveis os mesmos indicados anteriormente na Tabela 2, e uma única aeronave para 100 passageiros, com tempo mínimo de manutenção após voo de 45 minutos.

Tabela 5. Voos potenciais

\begin{tabular}{llll}
\hline Origem & Destino & Partida & Demanda \\
\hline A & B & $01: 40$ & 100 \\
A & C & $01: 40$ & 10 \\
C & A & $15: 00$ & 100 \\
\hline
\end{tabular}

Os voos foram assim definidos para criar um conflito para a escolha entre o voo de A para B ou o voo de A para C, ambos partindo às 01:40. A escolha mais direta, que independe do reposicionamento de aeronaves, é o voo de A para C. Entretanto, este é um voo longo (500 minutos), com uma demanda muito baixa, causando uma grande perda de demanda do voo de A para B. Esta situação faz com que a melhor alternativa seja realizar o voo de A para B, com um voo vazio, mais curto, de $\mathrm{B}$ para $\mathrm{C}$, e perdendo apenas a pequena demanda do voo de A para $\mathrm{C}$.

A geração do modelo leva menos de 1 segundo, resultando em modelo com 99 restrições e 167 variáveis. Sua resolução pelo CPlex não demorou mais que 0,03 s, e está representada na Tabela 6 , indicando exatamente a seleção dos voos de A para B, a criação do voo de B para $\mathrm{C}$ e a seleção do voo de C para A. Os eventos em itálico indicam voos de reposicionamento.

É possível notar, nesta solução, que um novo nó de pouso foi criado no aeroporto $\mathrm{C}$. Caso o aeroporto $\mathrm{C}$ fosse de operação restrita, isto é, operasse com o sistema de slots, isso não seria possível.

Pela tabela de voos potenciais, a chegada do voo de A para $\mathrm{C}$ ocorre às 10:00, definindo o slot de pouso. Seria inviável, portanto, o reposicionamento anterior, cujo pouso ocorre em C às 14:05, fazendo com que a solução ótima se- ja optar pelo voo de A para $\mathrm{C}$ e depois o voo de $\mathrm{C}$ para $\mathrm{A}$.

Para este caso, com restrição de operação no aeroporto $\mathrm{C}$, a criação do modelo leva menos de 1 segundo, resultando em um modelo com 34 restrições e 44 variáveis. Sua resolução pelo CPlex não demorou mais que $0,04 \mathrm{~s}$, e está representada na Tabela 7 , indicando exatamente a seleção de voos prevista.

Foram testadas variações destes testes, incluindo restrições de operação em alguns aeroportos, ou seja, envolvendo a criação de voos de reposicionamento usando slots de decolagem e pouso em aeroportos com operação restrita. Os modelos resultantes foram da mesma ordem de grandeza dos já apresentados, assim como os tempos de solução. Os resultados foram adequados, com arcos de reposicionamento sendo criados quando necessário e respeitando os horários dos slots pré-definidos.

\subsection{Conflito entre número de slots de pouso e decolagem}

Foram realizados também alguns testes com situações complexas hipotéticas, como a existência de aeroportos de operação restrita com dois slots de decolagem, mas apenas um de pouso, para avaliar o comportamento do modelo em tais situações.

O modelo permaneceu com dimensões similares aos anteriormente apresentados, com tempos de solução da mesma ordem, isto é, modelos gerados em poucos segundos e soluções obtidas em centésimos de segundo. As soluções encontradas foram compatíveis com a realidade, escolhendo o slot mais adequado em cada situação e mantendo vazios os slots inviáveis.

\section{APLICAÇÃO À MALHA DE UMA EMPRESA AÉREA BRASILEIRA}

Além dos testes de robustez, o modelo apresentado foi aplicado também em instâncias baseadas na malha aérea regional de uma empresa brasileira, considerando os dados disponibilizados pela empresa na Internet.

A malha tratada é composta por cinco cidades, com 104 voos semanais, sendo 20 voos diários de segunda a sexta e apenas dois voos de reposicionamento aos sábados e domingos.

Para cumprir os voos desta malha, são disponíveis três aeronaves modelo ATR-42/300 (AT43), com capacidade para 50 passageiros. Como a empresa trabalha com aviões de pequeno porte, o tempo de manutenção obrigatória entre voos é de apenas 15 minutos.

Tabela 6. Voos selecionados pelo modelo para uma aeronave de 100 passageiros

\begin{tabular}{llll}
\hline Frota (Aeronaves) & Evento & Origem (Dia - Hora) & Destino (Dia - Hora) \\
\hline 100 pax (1) & Voo & A $(0-01: 40)$ & B $(0-06: 40)$ \\
100 pax (1) & Voo & B $(0-07: 25)$ & C $(0-14: 05)$ \\
100 pax (1) & Voo & C $(0-15: 00)$ & A $(0-23: 20)$ \\
$-(0)$ & Voo & A $(0-01: 40)$ & C $(0-10: 00)$ \\
\hline
\end{tabular}

Tabela 7. Voos para a aeronave para 100 passageiros, com operação em C restrita

\begin{tabular}{llll}
\hline Frota (Aeronaves) & Evento & Origem (Dia - Hora) & Destino (Dia - Hora) \\
\hline 100 pax (1) & Voo & A $(0-01: 40)$ & C $(0-10: 00)$ \\
100 pax (1) & Voo & C $(0-15: 00)$ & A $(0-23: 20)$ \\
$-(0)$ & Voo & A $(0-01: 40)$ & B $(0-06: 40)$ \\
\hline
\end{tabular}


As instâncias geradas para análise foram divididas em 3 configurações, conforme especificadas a seguir.

- Configuração A: são problemas de cobertura na malha original da empresa, com a demanda de cada voo igual à capacidade das aeronaves utilizadas (frota homogênea);

- Configuração B: são problemas de programação de voos e alocação de frota, na malha original da empresa, porém considerando demandas médias por voo, com base nos dados de 2007 (ANAC, 2007); e

- Configuração C: são também problemas de programação de voos e alocação de frota e também consideram demandas médias para cada voo, mas neste caso considerou-se que a empresa obteve dois slots de pouso e dois de decolagem no aeroporto de Congonhas, às 9:00, 17:30, 10:00 e 18:30, respectivamente.

Cada uma destas configurações foi representada por problemas com diferentes composições de frotas de aeronaves, sempre para os 7 dias da semana, gerando resultados bastante variados com relação aos voos que devem ser executados pela empresa.

A Tabela 8 mostra as características dos modelos e tempos de soluções para as configurações $\mathrm{A}$ e B, sendo que o tempo de geração do modelo foi sempre inferior a $15 \mathrm{~s}$.

Nesses casos, a tabela de voos potenciais inclui todos os 104 voos da empresa. Para essas configurações, o modelo foi gerado sempre considerando 3 frotas com apenas uma aeronave em cada uma delas, proporcionando uma melhor visualização da solução. Com isso foi possível notar que não há diferenças significativas nos tempos de solução para aeronaves de características distintas.

A Tabela 9 apresenta os resultados obtidos com o processamento do modelo em cada uma destas configurações. Nessa tabela, cada valor representa o número de voos diários entre os aeroportos indicados na coluna A-B, separando-os em voos de ida $(\mathrm{A} \rightarrow \mathrm{B})$ e volta $(\mathrm{B} \rightarrow \mathrm{A})$.

Como é possível observar na configuração $\mathrm{A}$, as três aeronaves ATR-42/300 da empresa são suficientes para atender a todos os trechos. Por outro lado, essa configuração não representa uma situação realista, uma vez que a demanda de cada trecho foi considerada exatamente igual à capacidade de cada aeronave da frota.

As programações obtidas com a configuração $\mathrm{B}$, que considera a demanda média de cada trecho, baseada na demanda efetiva do ano de 2007, permitem verificar que o cumprimento de alguns trechos não é interessante com as aeronaves ATR-42/300. Esse resultado fica particularmente claro na configuração B.1, situação em que são alocadas apenas duas das três aeronaves do tipo ATR-42/300.

Como a demanda de alguns trechos é inferior à metade da capacidade das aeronaves consideradas na situação B.1 e, por essa razão, nenhuma aeronave foi alocada a eles, torna-se interessante avaliar o uso de aeronaves de menor capacidade para atender à demanda desses trechos.

Nesse sentido, a troca de uma das três aeronaves da empresa por uma aeronave menor, como o Embraer 140

Tabela 8. Características do modelo e tempos de solução para as configurações A e B

\begin{tabular}{lllll}
\hline Configuração: & $A$ & $B .1$ & $B .2$ & $B .3$ \\
\hline Frotas Disponíveis: & $3 x$ AT43 & $3 x$ AT43 & $2 x A T 43+E 140$ & $2 \times A T 43+E 120$ \\
\hline Frotas Alocadas & $3 \times$ AT43 & $2 \times$ AT43 & $2 \times$ AT43 + E140 & $2 \times$ AT43 + E120 \\
Variáveis & 35456 & 35456 & 35456 & 35456 \\
Restrições & 19526 & 19526 & 19526 & 19526 \\
Função Objetivo & 0 & $4,0 \times 10^{6}$ & $3,8 \times 10^{6}$ & $3,2 \times 10^{6}$ \\
Tempo de Solução (s) & 0,43 & 0,43 & 0,42 & 0,41 \\
\hline
\end{tabular}

Tabela 9. Resultados da alocação para as configurações A e B

\begin{tabular}{|c|c|c|c|c|c|c|c|c|c|}
\hline \multicolumn{2}{|c|}{ Configuração } & \multicolumn{2}{|c|}{$A$} & \multicolumn{2}{|c|}{ B.1 } & \multicolumn{2}{|l|}{ B.2 } & \multicolumn{2}{|l|}{ B.3 } \\
\hline \multicolumn{2}{|c|}{ Frota } & \multicolumn{2}{|c|}{$3 x$ AT43 } & \multicolumn{2}{|c|}{$2 x A T 43$} & \multicolumn{2}{|c|}{$2 x A T 43+E 140$} & \multicolumn{2}{|c|}{$2 x A T 43+E 120$} \\
\hline Dia & $A-B$ & $A \rightarrow B$ & $B \rightarrow A$ & $A \rightarrow B$ & $B \rightarrow A$ & $A \rightarrow B$ & $B \rightarrow A$ & $A \rightarrow B$ & $B \rightarrow A$ \\
\hline Dom & PLU-UBA & 1 & 0 & 1 & 0 & 1 & 0 & 1 & 0 \\
\hline Dom & UBA-UDI & 1 & 0 & 1 & 0 & 1 & 0 & 1 & 0 \\
\hline Seg & PLU-GVR & 2 & 2 & 2 & 2 & 2 & 2 & 2 & 2 \\
\hline Seg & PLU-IPN & 3 & 3 & 0 & 0 & 3 & 3 & 1 & 1 \\
\hline Seg & PLU-UDI & 4 & 2 & 2 & 2 & 1 & 2 & 4 & 2 \\
\hline Seg & UDI-UBA & 2 & 0 & 0 & 0 & 0 & 0 & 2 & 0 \\
\hline Seg & UBA-PLU & 2 & 0 & 0 & 0 & 0 & 0 & 2 & 0 \\
\hline Ter-Qui & PLU-GVR & 2 & 2 & 2 & 2 & 2 & 2 & 2 & 2 \\
\hline Ter-Qui & PLU-IPN & 3 & 3 & 0 & 0 & 3 & 3 & 1 & 1 \\
\hline Ter-Qui & PLU-UDI & 4 & 2 & 2 & 2 & 2 & 2 & 4 & 2 \\
\hline Ter-Qui & UDI-UBA & 2 & 0 & 0 & 0 & 0 & 0 & 2 & 0 \\
\hline Ter-Qui & UBA-PLU & 2 & 0 & 0 & 0 & 0 & 0 & 2 & 0 \\
\hline Sex & PLU-GVR & 2 & 2 & 2 & 2 & 2 & 2 & 2 & 2 \\
\hline Sex & PLU-IPN & 3 & 3 & 0 & 0 & 3 & 3 & 1 & 1 \\
\hline Sex & PLU-UDI & 4 & 2 & 1 & 2 & 4 & 2 & 4 & 2 \\
\hline Sex & UDI-UBA & 2 & 0 & 0 & 0 & 2 & 0 & 2 & 0 \\
\hline Sex & UBA-PLU & 2 & 0 & 0 & 0 & 2 & 0 & 2 & 0 \\
\hline Sáb & UDI-UBA & 1 & 0 & 0 & 0 & 0 & 0 & 1 & 0 \\
\hline Sáb & UBA-PLU & 1 & 0 & 0 & 0 & 0 & 0 & 1 & 0 \\
\hline
\end{tabular}


(E140, para 44 passageiros) ou o Embraer 120 (E120, para 30 passageiros), faria com que a cobertura fosse bastante próxima da total, mantendo o equilíbrio entre demanda e oferta, como pode ser visto pelas configurações B.2 e B.3, respectivamente.

A Tabela 10 mostra as características dos modelos e tempos de soluções para a configuração $\mathrm{C}$, com tempo de geração do modelo sempre inferior a $35 \mathrm{~s}$. Nesses casos, a tabela de voos potenciais é composta por 164 voos, incluindo os novos voos para Congonhas. Para essas configurações, os modelos gerados consideraram números diferentes de frotas, cada uma delas com uma única aeronave.

A Tabela 11 apresenta os resultados obtidos com o processamento do modelo em cada uma destas configurações. Também nessa tabela os valores representam o número de voos entre os aeroportos indicados na coluna A-B, separando-os em voos de ida $(\mathrm{A} \rightarrow \mathrm{B})$ e volta $(\mathrm{B} \rightarrow \mathrm{A})$.

Como é possível observar na Tabela 11, na configuração C.1 a frota original da empresa não é capaz de cobrir todos os voos propostos nesse caso. Há um deslocamento das ae- ronaves para cumprir os voos que envolvem Congonhas, com maior demanda e duração do voo, substituindo diversos voos que ocorriam anteriormente.

A troca de um ATR-42/300 por uma aeronave maior, como o Embraer 170 (E170, 70 passageiros), não altera o número de voos, mas indica um melhor equilíbrio da oferta/demanda, percebido pela redução do valor da função objetivo, como pode ser visto na configuração C.2.

A adição de uma aeronave como o Embraer 120 à configuração anterior, por outro lado, melhora a cobertura e amplia o número de voos, como pode ser verificado na configuração C.3.

Já na configuração C.4, a adição de uma quinta aeronave, outro Embraer 120, permite cobertura total - excluindo-se apenas os voos impossíveis - de e para Congonhas, uma vez que os slots foram ocupados com voos entre Belo Horizonte (PLU) e Congonhas (CGH).

\subsection{Análise dos Resultados}

Os resultados dos testes e da aplicação indicam dois pontos de relevância com relação à aplicabilidade do modelo.

Tabela 10. Características do modelo e tempos de solução para a configuração C

\begin{tabular}{lllll}
\hline Configuração: & $C .1$ & $C .2$ & $C .3$ & $C .4$ \\
\hline Frotas Disponíveis: & $3 x A T 43$ & $2 x A T 43+E 170$ & $E 120+2 x A T 43+E 170$ & $2 x E 120+2 x A T 43+E 170$ \\
\hline Frotas Alocadas & $3 \times A T 43$ & $2 x$ AT43+E170 & E120+2xAT43+E170 & 2xE120+2xAT43+E170 \\
Variáveis & 45053 & 45053 & 60016 & 74979 \\
Restrições & 24286 & 24286 & 32320 & 40354 \\
Função Objetivo & $16,1 \mathrm{mi}$ & $14,5 \mathrm{mi}$ & $14,4 \mathrm{mi}$ & $14,4 \mathrm{mi}$ \\
Tempo de Solução (s) & 0,91 & 0,61 & 0,95 & 10,4 \\
\hline
\end{tabular}

Tabela 11. Resultados da alocação para a configuração $C$

\begin{tabular}{|c|c|c|c|c|c|c|c|c|c|}
\hline \multicolumn{2}{|c|}{ Configuração } & \multicolumn{2}{|l|}{ C.1 } & \multicolumn{2}{|l|}{ C.2 } & \multicolumn{2}{|l|}{ C.3 } & \multicolumn{2}{|l|}{ C.4 } \\
\hline \multicolumn{2}{|c|}{ Frota } & \multicolumn{2}{|c|}{$3 x$ AT43 } & \multicolumn{2}{|c|}{$2 x A T 43+E 170$} & \multicolumn{2}{|c|}{$E 120+2 x A T 43+E 170$} & \multicolumn{2}{|c|}{$2 x E 120+2 x A T 43+E 170$} \\
\hline Dia & $A-B$ & $A \rightarrow B$ & $B \rightarrow A$ & $A \rightarrow B$ & $B \rightarrow A$ & $A \rightarrow B$ & $B \rightarrow A$ & $A \rightarrow B$ & $B \rightarrow A$ \\
\hline Dom & PLU-UBA & 1 & 0 & 1 & 0 & 1 & 0 & 1 & 0 \\
\hline Dom & UBA-UDI & 1 & 0 & 1 & 0 & 1 & 0 & 1 & 0 \\
\hline Seg & PLU-CGH & 2 & 2 & 2 & 2 & 2 & 2 & 2 & 2 \\
\hline Seg & PLU-GVR & 2 & 2 & 2 & 2 & 2 & 2 & 2 & 2 \\
\hline Seg & PLU-IPN & 0 & 0 & 0 & 0 & 1 & 1 & 3 & 3 \\
\hline Seg & PLU-UDI & 2 & 2 & 2 & 2 & 4 & 2 & 4 & 2 \\
\hline Seg & UDI-CGH & 0 & 0 & 0 & 0 & 0 & 0 & 0 & 0 \\
\hline Seg & UDI-UBA & 0 & 0 & 0 & 0 & 2 & 0 & 2 & 0 \\
\hline Seg & UBA-PLU & 0 & 0 & 0 & 0 & 2 & 0 & 2 & 0 \\
\hline Seg & UBA-CGH & 0 & 0 & 0 & 0 & 0 & 0 & 0 & 0 \\
\hline Ter-Qui & PLU-CGH & 2 & 2 & 2 & 2 & 2 & 2 & 2 & 2 \\
\hline Ter-Qui & PLU-GVR & 2 & 2 & 2 & 2 & 2 & 2 & 2 & 2 \\
\hline Ter-Qui & PLU-IPN & 0 & 0 & 0 & 0 & 1 & 1 & 3 & 3 \\
\hline Ter-Qui & PLU-UDI & 2 & 2 & 2 & 2 & 4 & 2 & 4 & 2 \\
\hline Ter-Qui & UDI-CGH & 0 & 0 & 0 & 0 & 0 & 0 & 0 & 0 \\
\hline Ter-Qui & UDI-UBA & 0 & 0 & 0 & 0 & 2 & 0 & 2 & 0 \\
\hline Ter-Qui & UBA-PLU & 0 & 0 & 0 & 0 & 2 & 0 & 2 & 0 \\
\hline Ter-Qui & UBA-CGH & 0 & 0 & 0 & 0 & 0 & 0 & 0 & 0 \\
\hline Sex & PLU-CGH & 2 & 2 & 2 & 2 & 2 & 2 & 2 & 2 \\
\hline Sex & PLU-GVR & 2 & 2 & 2 & 2 & 2 & 2 & 2 & 2 \\
\hline Sex & PLU-IPN & 0 & 0 & 0 & 0 & 1 & 1 & 3 & 3 \\
\hline Sex & PLU-UDI & 1 & 2 & 2 & 2 & 4 & 2 & 4 & 2 \\
\hline Sex & UDI-CGH & 0 & 0 & 0 & 0 & 0 & 0 & 0 & 0 \\
\hline Sex & UDI-UBA & 0 & 0 & 0 & 0 & 2 & 0 & 2 & 0 \\
\hline Sex & UBA-PLU & 0 & 0 & 0 & 0 & 2 & 0 & 2 & 0 \\
\hline Sex & UBA-CGH & 0 & 0 & 0 & 0 & 0 & 0 & 0 & 0 \\
\hline Sáb & UDI-UBA & 0 & 0 & 0 & 0 & 1 & 0 & 1 & 0 \\
\hline Sáb & UBA-PLU & 0 & 0 & 0 & 0 & 1 & 0 & 1 & 0 \\
\hline
\end{tabular}


O primeiro deles refere-se à adequação dos resultados à prática, isto é, os resultados são aplicáveis e, dentro das considerações modeladas, propiciam a escolha dos melhores voos para o conjunto de aeronaves de uma empresa. $\mathrm{O}$ valor da função objetivo cresce à medida que cresce a disparidade entre o número de assentos e a demanda por estes assentos, permitindo uma comparação entre soluções que envolvam inclusive conjuntos de aeronaves distintas, desde que sejam mantidos o conjunto de voos potenciais e as demandas previstas. Como consequência, este valor pode ser considerado como um indicador na verificação do impacto ocasionado pelo uso de novas aeronaves ou, ainda, pela imposição da realização de um dado voo.

O segundo ponto relevante refere-se aos tempos de processamento, que sofre um impacto significativo com o crescimento do número de frotas, uma vez que cada frota é representada por uma rede diferente. O tempo de processamento cresce de maneira não linear com relação ao crescimento do número de variáveis, como é possível verificar nas rodadas da configuração $\mathrm{C}$ (notadamente entre C.3 e as demais configurações), onde não ocorrem mudanças na tabela de voos potenciais nem na demanda, apenas no número de frotas disponíveis. Por outro lado, os tempos permanecem gerenciáveis, mesmo na situação de 5 frotas distintas, para a malha regional considerada.

\section{CONCLUSÕES}

Este trabalho apresentou um modelo que permite a determinação de soluções integradas para os problemas de Alocação de Frota e de Programação de Voos, considerando características e particularidades da operação aeroportuária.

Um modelo de programação linear inteira associado a uma formulação em rede espaço-tempo foi proposto, testado e avaliado em situações específicas, e aplicado com sucesso a instâncias relacionadas a um caso real de empresa aérea regional brasileira.

O modelo desenvolvido exige, para sua aplicação, o conhecimento da demanda potencial para cada voo candidato e a disponibilidade de aeronaves de cada tipo. Os testes e aplicações mostraram resultados compatíveis com a realidade, com baixos tempos de processamento, mesmo considerando a existência de várias frotas distintas, pelo menos no caso de empresas aéreas operando regionalmente.

Extensões do modelo estão sendo consideradas, incorporando pesos diferenciados para assentos vazios e demanda perdida, bem como restrições de operação de determinados tipos de aeronaves em alguns aeroportos e redistribuição da demanda entre diversos voos concorrentes.

\section{AGRADECIMENTOS}

Os autores agradecem à CAPES (Coordenação de Aperfeiçoamento de Pessoal de Nível Superior), ao CNPq (Conselho Nacional de Desenvolvimento Científico e Tecnológico) e ao LPT/EPUSP (Laboratório de Planejamento e Operação de Transportes da EPUSP).

\section{REFERÊNCIAS BIBLIOGRÁFICAS}

Abara, J. (1989) Applying Integer Linear Programming to the Fleet Assignment Problem. Interfaces, v.19, n.4, p.21-28.

ANAC (2007) Anuário Estatístico da Agência Nacional de Aviação Civil vol.I. Disponível em: <http://www2.anac.gov.br/estatistica/ anuarios.asp $>$. (Acesso em 16/03/2011).
ANAC (2008) ANAC cria regra de slots para aumentar concorrência em aeroportos saturados. Disponível em: <http://www2.anac.gov.br/ imprensa/AnacCriaRegraDeSlots011008.asp $>$. (Acesso em 16/03/2011).

Barnhart, C.; A. M. Cohn; E. L. Johnson; D. Klabjan; G. L. Nemhauser e P. H. Vance (2003) Airline Crew Scheduling in Handbook of Transportation Science. 2nd ed. Kluwer's International Series.

Caetano, D. J. e N. D. F. Gualda (2008) Modelagem do Problema de Alocação de Frota de uma Empresa Aérea Brasileira. Anais do XXII Congresso de Pesquisa e Ensino em Transportes, ANPET, Fortaleza, v. 1, p. 2173-2176.

CGNA (2009) Capacidades dos Aeroportos. Disponível em: $<$ http://www.cgna.gov.br/aeroportos/aeroportos.htm>. (Acesso em 10/07/2009).

Dantzig, G. B. (1974) Linear programming and extensions. New Jersey: Princeton University Press.

Dantzig, G.B e D.R. Fulkerson (1954) Minimizing the number of carriers to meet a fixed schedule, Naval Res. Log. v. 1.

Gomes, W. P. e N. D. F. Gualda (2008) Otimização da Formação de Viagens no Processo de Alocação de Tripulantes e Aeronaves (Crew Pairing Problem). Anais do XXII Congresso de Pesquisa e Ensino em Transportes, ANPET, Fortaleza, v. 1, p. 1010-1020.

Hane, C.; C. Barnhart; E. Johnson; R. Marsten; G. Nemhauser e G. Sigismondi (1994) A fleet assignment problem: Solving a large-scale integer program, Technical report, Georgia Institute of Technology, School of Industrial and System Engineering. Report Series 92-04.

Klabjan, D. (2004) Large-scale models in the airline industry, in G. Desaulniers, J. Desroriers, M. M. Solomon, editors, Column Generation, Kluwer Academic Publishers.

MD (2008) Congonhas voltará a ter escalas e conexões. Disponível em: $<$ https://www.defesa.gov.br/index.php/noticias-anteriores-do$\mathrm{md} / 31830-21-01-2008$---defesa---congonhas-voltara-a-terescalas-e-conexoes.html>. (Acesso em 16/03/2011).

Oliveira, A. (2009) Transporte Aéreo - Economia e Políticas Públicas. Pezco Editora. São Paulo.

Rabetanety, A.; J. Calmet e C. Schoen (2006) Airline Schedule Planning Integrated Flight Schedule Design and Product Line Design. Tese (doutorado). Universität Karlsruhe. Karlsruhe.

Sherali, H.D; E. K. Bish; X. Zhu (2006) Airline fleet assignment concepts, models, and algorithms, European Journal of Operational Research, v. 172, n. 1, p. 1-30.

Swan, W.M. (2002) Airline route developments: a review of history. Journal of Air Transport Management. v. 8, n. 5. p. 349-353.

Swan, W.M.; N. Adler. (2006) Aircraft trip cost parameters: A function of stage length and seat capacity. Transportation Research Part E: Logistics and Transportation Review, v. 42, n. 2, p. 105-115. 